\title{
Ergebnisse einer strukturierten Diagnostik bei Patienten mit chronischer Dyspnoe in zwei kardiologischen Facharztpraxen
}

\author{
Results of a Structural Diagnosis of Patients with Chronic Dyspnoea \\ Recruited from Two Cardiological Practices
}

Autoren

Institute
R. Ewert ${ }^{1}$, A. Obst ${ }^{1}$, C. Bahr ${ }^{2}$, C. Weirich ${ }^{2}$, F. Henschel ${ }^{3}$, A. Rink ${ }^{3}$

${ }^{1}$ Universitätsmedizin Greifswald, Klinik für Innere Medizin B (Direktor: Prof. Dr. med. S. B. Felix)

${ }^{2}$ Gemeinschaftspraxis Innere Medizin/Kardiologie Pasewalk

3 Kardiologische Gemeinschaftspraxis Rostock

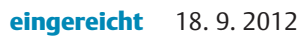

akzeptiert 15.10. 2012

\section{Bibliografie}

DOI http://dx.doi.org/

10.1055/s-0032-1325905

Online-Publikation: 16.11 .2012

Pneumologie 2013; 67: 35-39

(c) Georg Thieme Verlag KG

Stuttgart · New York

ISSN 0934-8387

\section{Korrespondenzadresse Prof. Dr. med. Ralf Ewert}

Ernst-Moritz-Arndt-Universität Greifswald, Universitätsmedizin Klinik für Innere Medizin B, Bereich Pneumologie/ Infektiologie Fleischmannstraße 8 17475 Greifswald ewert@uni-greifswald.de

\section{Zusammenfassung \\ $\nabla$}

Während zur Diagnostik der Ursachen bei akuter Dyspnoe gut validierte Algorithmen vorliegen, fehlen solche allgemein gültigen Handlungsanweisungen bei Patienten mit chronischer Dyspnoe. In der vorliegenden Arbeit wurde ein selbstentwickeltes mehrstufiges Vorgehen bei der Diagnostik von 246 Patienten mit chronischer Dyspnoe in zwei kardiologischen Praxen angewendet. Neben der anamnestischen und klinischen Untersuchung beinhaltet es verschiedene apparative Verfahren, welche im ambulanten Bereich verfügbar sind. Im Ergebnis konnte gezeigt werden, dass bei über $50 \%$ der Patienten mit chronischer Dyspnoe die Ursache mittels anamnestischer und klinischer Untersuchung sowie Durchführung eines Elektrokardiogramms und einer transthorakalen Echokardiografie nach Ansicht der Untersucher ausreichend geklärt werden kann. Die zusätzliche Einbeziehung der Ergebnisse der Bodyplethysmografie und einer kapillären Blutgasanalyse erlaubte in über $80 \%$ der Patienten eine ausreichende Klärung der Ursache der chronischen Dyspnoe. Die in einem weiteren Schritt durchgeführte Spiroergometrie konnte bei weiteren etwa 10\% der Fälle die Dyspnoe abklären. Grenzwerte lungenfunktioneller Parameter, die zur Differenzierung der Ursachen einer akuten Dyspnoe verwendet werden, scheinen zur Abklärung bei Patienten mit chronischer Dyspnoe nicht geeignet. Mittels eines einfachen abgestuften diagnostischen Algorithmus konnte bei über 95\% der Patienten mit chronischer Dyspnoe die Ursache unter ambulanten Bedingungen ausreichend abgeklärt werden.

\section{Abstract \\ $\nabla$}

While well validated algorithms exist for the diagnosis of patients with acute dyspnoea, such generally valid procedural instructions are lacking for patients with chronic dyspnoea. The diagnostic approach presented here is based on investigations of 246 patients with chronic dyspnoea recruited from two cardiological practices using a self-developed multi-level procedure. Besides anamnestic and clinical examinations, different diagnostic procedures, available in the ambulant range, are included. The results suggest that in over $50 \%$ of the cases the cause of chronic dyspnoea can be determined from anamnestic and clinical examinations as well as from electrocardiogram and echocardiography. Additional inclusion of lung function and capillary blood gas analysis permitted a sufficient clarification in over $80 \%$ of the cases. In a further step, cardiopulmonary exercise testing clarified the reasons for chronic dyspnoea in approximately $10 \%$ of the remaining patients. Threshold values of lung function parameters as used for the differentiation of acute dyspnoea, do not seem suitable for the diagnosis of patients with chronic dyspnoea. By means of a simple step-wise diagnostic algorithm, applicable under ambulant conditions, the cause of chronic dyspnoea could sufficiently be clarified in more than $95 \%$ of the cases. 


\section{Einleitung}

Eine Vielzahl von Erkrankungen und Störungen führt bei Patienten zur Wahrnehmung einer chronischen Dyspnoe. Ungeachtet der verschiedenen Definitionen wird die Dyspnoe überwiegend „als ein subjektives Missempfinden im Zusammenhang mit der Atmung“ definiert [1]. Seit Jahrzehnten existieren Bemühungen zur standardisierten Erfassung und Quantifizierung der akuten und chronischen Dyspnoe, wobei das Spektrum von visuellen Analogskalierungen bis hin zur Beschreibung im Rahmen von mehrdimensionalen Fragebögen zur Lebensqualität reicht [2-4]. Dabei gilt es u.a. die verschiedenen Aspekte der Dyspnoe (sensorische Intensität und Qualität; affektive Intensität als Grad des Missempfindens und den kognitiven Aspekt der emotionalen Reaktion) zu erfassen [5].

Das diagnostische Vorgehen wird wesentlich dadurch bestimmt, ob eine neu aufgetretene (ggf. akut beginnende) oder eine chronische (ggf. mit bekannter Ursache) Dyspnoe vorliegt. Es existieren gut etablierte Algorithmen zur validen Diagnostik der akuten Dyspnoe [6]. Zur Abklärung der Ursachen einer chronischen Dyspnoe fehlen vergleichbare, krankheitsübergreifende Handlungsanweisungen. Daher wird im ersten Schritt meist versucht, die vordergründig zugrundeliegende Störung zu erfassen (pulmonale, kardiale oder sonstige Erkrankung). Insbesondere bei Vorliegen mehrerer möglicher Ursachen für eine Dyspnoe sind zur Differenzierung des Anteils der einzelnen Ursachen kardiopulmonale Belastungsuntersuchungen sinnvoll, wobei die Spiroergometrie für diese Frage besonders gut etabliert ist [7]. Ungeachtet des breiten Spektrums somatischer Störungen und Erkrankungen sollten auch die psychosomatischen Aspekte der Dyspnoe ausreichend gewürdigt und psychische Störungen in die Differenzialdiagnose einbezogen werden [8].

Ziele der vorliegenden Arbeit waren a) die Erfassung der Ursachen einer chronischen Dyspnoe bei konsekutiven Patienten in zwei kardiologischen Praxen mittels eines strukturierten diagnostischen Algorithmus und b) die Überprüfung von Parametern zur Differenzierung der akuten Dyspnoe auf ihre Anwendbarkeit bei Patienten mit chronischer Dyspnoe.

\section{Methodik}

In zwei Facharztpraxen für Innere Medizin/Kardiologie $(1 \times$ Stadt mit $>200.000$ Einwohnern und $1 \times$ Stadt mit 11.000 Einwohnern und ländlichem Umfeld) wurden mit Beginn des II. Quartals 2010 fortlaufend Patienten mit einer chronischen Dyspnoe (d. h. länger als zwei Wochen bestehend) zur Teilnahme an dieser Studie eingeladen. Nach Aufklärung über die Zielstellung der Studie mit nachfolgender Datenerhebung wurde das Einverständnis der Patienten dokumentiert.

Zur Durchführung der Studie existiert ein Votum der Ethikkommission der Ernst-Moritz-Arndt Universität Greifswald vom 26.01.2010 (Reg.-Nr. BB 03/10).

\section{Patienten \\ $\nabla$}

Es wurden 246 Patienten (mittleres Alter 67,8 \pm 11 Jahre) in die Untersuchung eingeschlossen. Die chronische Dyspnoe bestand im Mittel seit 11,9 \pm 14,2 Jahren. Bei 22,0\% der Patienten wurde der Schweregrad der Luftnot (nach der modifizierten MRC (Medical Research Council)- Skalierung) als „leicht“ (Grad 1), bei $61 \%$ als
Tab. 1 Demografische Daten der ausgewerteten Patienten.

\begin{tabular}{|l|l|}
\hline Charakteristik & $\mathbf{N}=\mathbf{2 4 6}$ \\
\hline Alter (in Jahren) & $67,8 \pm 11,0$ \\
\hline Luftnot seit (in Monaten) & $11,9 \pm 14,2$ \\
\hline Raucher (\%) & $73,6(n=181)$ \\
\hline Nie-Raucher & $1,2(n=3)$ \\
\hline Gelegentlich & $11,0(n=27)$ \\
\hline Raucher & $14,2(n=35)$ \\
\hline Ex-Raucher & $88,5 \pm 89,2$ \\
\hline Ex-Raucher seit (in Monaten) & \\
\hline Packungsjahre & $29,3 \pm 16,8$ \\
\hline Raucher & $28,0 \pm 18,7$ \\
\hline Ex-Raucher & \\
\hline Komorbiditäten (nach Charlson-Index in \%) & $44,3(n=101)$ \\
\hline 0 & $30,3(n=69)$ \\
\hline 1 & $16,2(n=37)$ \\
\hline 2 & $3,1(n=7)$ \\
\hline 3 & $2,2(n=5)$ \\
\hline 4 & $2,6(n=6)$ \\
\hline 5 & $0,9(n=2)$ \\
\hline 6 & $0,4(n=1)$ \\
\hline 9 & \\
\hline Grad der Dyspnoe (nach ATS Scale in \%) & $22,0(n=54)$ \\
\hline 1 & $60,9(n=150)$ \\
\hline 2 & $17,1(n=42)$ \\
\hline 3 & \\
\hline
\end{tabular}

„mäßig“ (Grad 2) und bei 17,0\% als „schwer“ (Grad 3) eingeschätzt. 73,6\% der Patienten waren „Nie-Raucher“, 14,2\% waren „Ex-Raucher“ und 11\% waren „Raucher“. Bei 44,3\% der Patienten lagen keine relevanten Ko-Morbiditäten (Erfassung anhand des Charlson-Index, 9) vor ( Tab.1).

\section{Untersuchungsprotokoll}

$\nabla$

Bei allen Patienten wurden neben der anamnestischen und klinischen Datenerhebung eine apparative Basisuntersuchung mittels Elektrokardiogramm und transthorakaler Echokardiografie durchgeführt (Schritt 1). Im Vorfeld der Studie wurde festgelegt, dass bei Vorliegen a) einer eingeschränkten linksventrikulären systolischen Funktion (Simpson-Methode mit LVEF <50\%), b) einer relevant gestörten linksventrikulären diastolischen Funktion (Anwendung Tissue-Doppler E/E'<10 bzw. Dopplermessung des Mitraleinstroms $0,75<\mathrm{E} / \mathrm{A}<1,5$ und nach Valsalva Manöver $\Delta E / A>0,5), c)$ dem Nachweis eines Aorten- bzw. Mitralklappenvitiums $>1$. Grades, d) der Erfassung eines Vorhofflimmerns, e) einer Wandbewegungsstörung (außer alleinig septal) oder f) einer pulmonalen Hypertonie (Regurgitationsgeschwindigkeit an der Trikuspidalklappe von mehr als 3,4 m/s) die Ursache der chronischen Dyspnoe als „ausreichend geklärt“ angesehen wird. Sofern keine ausreichende Erklärung für die Ursache der chronischen Dyspnoe mit den genannten Untersuchungen erfasst werden konnte, wurden weitere apparative Methoden zur Anwendung gebracht (in Zusammenarbeit mit kooperierenden Kollegen). Diese waren eine Bodyplethysmografie, einschließlich Messung der Diffusionskapazität sowie eine kapilläre Blutgasanalyse (Schritt 2). Im Vorfeld wurde festgelegt, dass die Dyspnoe als ausreichend erklärt galt, wenn a) eine restriktive (TLC $<80 \%$ der Norm) oder obstruktive (FEV1/VC $<70 \%$ ) Ventilationsstörung vorlag bzw. b) ein Asthma bronchiale bekannt (bzw. wahrscheinlich) war. 
Wenn nach den ersten beiden Schritten der Diagnostik keine ausreichende Erklärung für die Ursache der chronischen Dyspnoe nachzuweisen war, erfolgte die Durchführung einer Spiroergometrie nach einem modifizierten Jones-Protokoll ([10], Schritt 3).

Zudem waren unabhängig vom Studienprotokoll weitere Untersuchungen im Ermessen der betreuenden Kollegen/innen möglich.

Im Ergebnis der strukturierten Diagnostik wurde der Grad der chronischen Dyspnoe (nach der modifizierten MRC (Medical Research Council)-Skalierung [11]) dokumentiert und ein ärztliches Urteil zur Ursache der chronischen Dyspnoe abgegeben („ausreichend geklärt“ bzw. „nicht ausreichend geklärt“). Bei ausreichender Klärung wurde eine Zuordnung der Ursache der chronischen Dyspnoe als „kardial“, „pulmonal“ bedingt bzw. „Sonstige“ (Dekonditionierung, kombinierte Störungen) vorgenommen.

In einem zweiten Schritt wurde geprüft, ob in der Literatur verwendete Parameter [13-15] zur Differenzierung der Ursachen einer akuten Dyspnoe ggf. auch für die Differenzierung der Ursachen einer chronischen Dyspnoe geeignet sind. Diese Parameter sind einfach zu erhebende lungenfunktionelle Werte (PEF, peak expiratory flow), teilweise in Kombination mit Werten der kapillären Blutgasanalyse (Dypnoe-Differentiation-Index, DDI als berechneter Wert aus $\left.\mathrm{PEF}[\mathrm{L} / \mathrm{min}] \times \mathrm{PaO}_{2}[\mathrm{mmHg}] / 1000\right)$.

\section{Datenbearbeitung}

Sämtliche Ergebnisse der strukturierten Diagnostik wurden in Form einer Datenbank (Microsoft Excel ${ }^{\circledR}$ ) erfasst. Kontinuierliche Daten wurden als Mittelwert und Standardabweichung, Nominaldaten in Prozent angegeben. ROC-Kurven für ausgewählte Parameter wurden erstellt und die Fläche unter der Kurve (AUC) berechnet. Für einige Parameter wurde der Cut-Off-Wert aus dem maximalen Produkt aus Sensitivität und Spezifität bestimmt und als optimaler Cut-Off-Wert für eine Prognose angesehen. Ein p-Wert von<0,05 wurde als signifikant erachtet. Alle statistischen Analysen erfolgten mit den Programmen SAS 9.1 (SAS Institute Inc., Cary, NC, USA) und R (Statistik-Paket).

\section{Ergebnisse}

Von den Patienten konnten nach anamnestischer, klinischer und apparativer Untersuchung im Rahmen der Vorstellung beim Kardiologen in 141/246 (d.h. 57,3\%) der Fälle die Ursache der chronischen Dyspnoe ausreichend geklärt und mit dem Urteil „kardial bedingt“ $(n=126)$ versehen werden. In weiteren 15 Fällen wurde die Ursache der Dyspnoe als „Sonstige“ (kombinierte pulmonale/kardiale Störung [ $\mathrm{n}=13]$; klinisch manifeste Depression $[\mathrm{n}=2]$ ) eingeordnet.

Somit blieben nach Durchführung der Basisdiagnostik (Schritt 1) 105/246 (d.h. 42,7\%) der Patienten ohne ausreichende Klärung der Ursache für die vorliegende chronische Dyspnoe.

Zur weiteren Abklärung wurden dann bei 102/105 Patienten (drei Patienten sind ohne Angabe von Gründen nicht mehr erschienen) zusätzliche Untersuchungen durchgeführt (Schritt 2). Im Ergebnis konnte dann bei 60 Patienten eine ausreichende Klärung erfolgen, und es wurde eine „pulmonale“ Ursache der chronischen Dyspnoe angenommen.

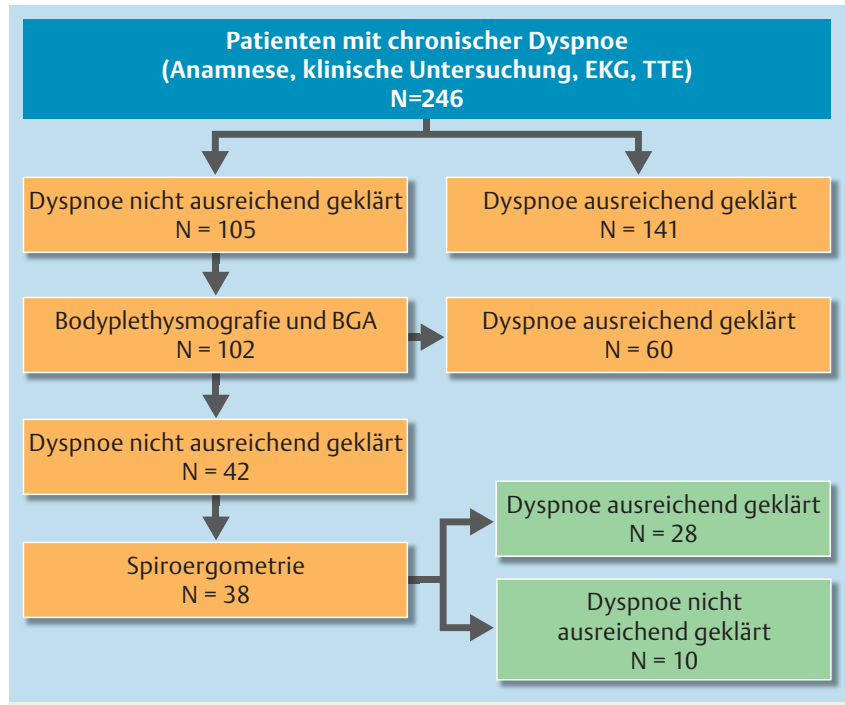

Abb.1 Abklärung der Ursachen einer chronischen Dyspnoe.

Somit blieb auch nach Durchführung der ersten beiden Schritte der Basisdiagnostik die Ursache der chronischen Dyspnoe bei 42/102 (d.h. 41,2\%) Patienten weiter „nicht ausreichend geklärt“. Im Rahmen der Stufendiagnostik wurde dann bei 38/42 Patienten (vier Patienten sind ohne Angabe von Gründen nicht erschienen) eine Spiroergometrie durchgeführt. Im Ergebnis konnte bei 28/38 Fällen die Ursachen der chronischen Dyspnoe ausreichend geklärt werden („kardial“ [n=9], „Sonstige“ [ $n=19$, wobei in 16 Fällen „Dekonditionierung“ und in 3 Fällen „kombinierte pulmonal/kardiale Störung“" angenommen wurde]).

Neben den genannten apparativen Methoden im Rahmen der Stufendiagnostik wurden zur Abklärung der Ursache ihrer Beschwerden weitere Untersuchungen durchgeführt, welche nicht Bestandteil des Untersuchungsplanes waren und somit alleinig durch die betreuenden Kollegen/innen indiziert wurden. In 14 Fällen erfolgte eine Untersuchung mittels Linksherzkatheter, wobei in drei Fällen eine koronare Herzerkrankung diagnostiziert wurde. In 12 Fällen (davon zwei mit pathologischem Ergebnis) wurden kardiale SPECT-Untersuchungen durchgeführt und in drei weiteren Fällen eine thorakale Computertomografie (in einem Fall Nachweis eines Aortenaneurysmas). In vier Fällen erfolgte eine Röntgen-Thorax-Aufnahme, jeweils mit unauffälligem Befund.

Somit konnte letztlich nach Durchführung einer strukturierten, mehrstufigen Diagnostik bei nur 10/239 (4,2\%) Patienten (sieben Patienten haben nicht alle drei Stufen der Diagnostik erhalten und wurden daher bei der Betrachtung nicht berücksichtigt) die Ursache der chronischen Dyspnoe nach Ansicht der betreuenden Ärzte nicht „ausreichend geklärt“ werden ( $\bullet$ Abb. 1).

Bei zwei dieser Patienten erfolgte eine Myokardszintigrafie mit unauffälligem Befund, bei einem Patienten wurde ein Linksherzkatheter mit unauffälligem Befund durchgeführt, bei zwei Patienten wurde die Durchführung eines Rechtsherzkatheters empfohlen, jedoch von den Patienten abgelehnt. Fünf Patienten sind zur vereinbarten weiterführenden Untersuchung nicht erschienen.

Die Überprüfung der Anwendbarkeit ausgewählter Parameter zur Differenzierung der akuten Dyspnoe bei unserem Patientengut mit chronischer Dyspnoe erfolgte nur bei Patienten mit Zuordnung zu „pulmonalen“ bzw. „kardialen“ Ursachen der Dyspnoe und vollständig vorhandenen Werten. Bei Verwendung des 

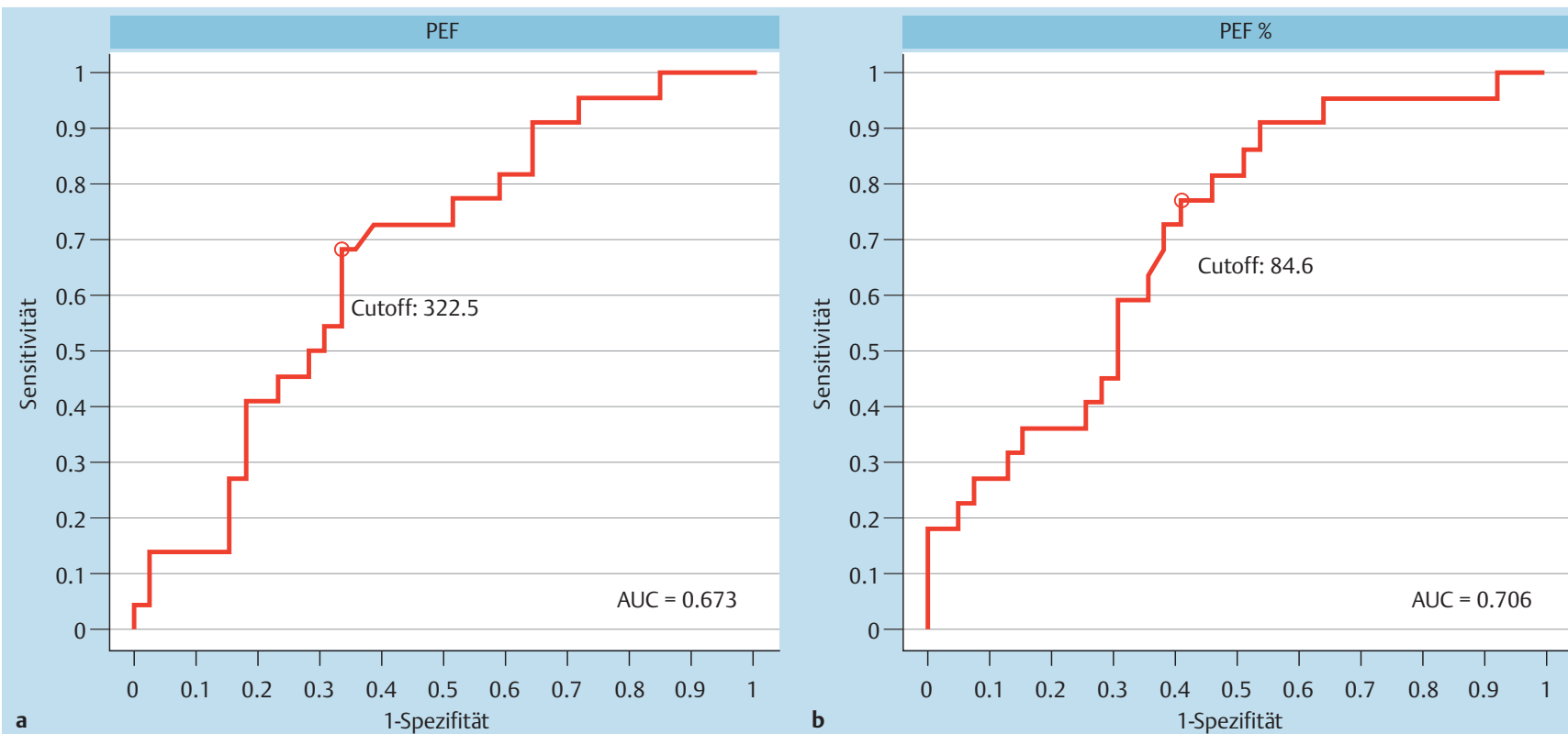

Abb.2 ROC-Kurven, AUC und optimale Grenzwerte zur Differenzierung der Patienten mit chronischer Dyspnoe. Links (a) unter Verwendung des Nominalwertes für den PEF-Wert und rechts (b) unter Verwendung des prozentualen Normwertes für den PEF-Wert.

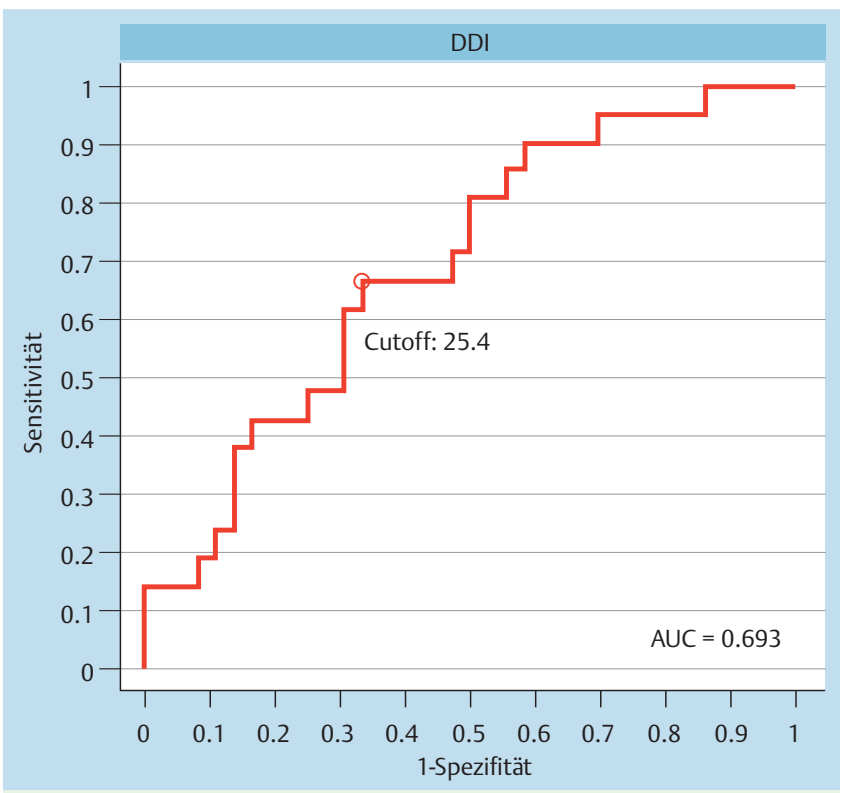

Abb.3 ROC-Kurve, AUC und optimaler Grenzwert zur Differenzierung der Patienten mit chronischer Dyspnoe anhand des DDI-Wertes.

PEF-Wertes (Nominalwert, $\mathrm{n}=61$ ) konnte ein Wert von 322 Liter/ min mit einer Sensitivität von 68\% und Spezifität von 67\% eine „kardiale“ von einer „pulmonalen“ Ursache der chronischen Dyspnoe differenzieren. Wenn man den PEF-Wert als prozentualen Wert der Norm verwendet, differenziert ein Wert von $85 \%$ der Norm mit einer Sensitivität von 77\% und Spezifität von 59\% zwischen den beiden Ursachen der Dyspnoe ( $\bullet$ Abb.2a,b). Unter Verwendung des Dyspnoe-Differentiation-Index (DDI, $\mathrm{n}=57$ ) konnte ein Wert von 25 mit einer Sensitivität von 68\% und einer Spezifität von $67 \%$ zwischen einer „pulmonalen“ bzw. „kardialen“ Ursache der chronischen Dyspnoe unterscheiden ( $\bullet$ Abb.3).

\section{Diskussion \\ $\nabla$}

Zur Differenzierung der Ursachen einer plötzlichen Dyspnoe existiert eine Reihe von diagnostischen Algorithmen, wobei diese vorrangig anhand von Patienten aus Notaufnahmen von Krankenhäusern validiert wurden [12-15]. Allgemeine Handlungsempfehlungen zum diagnostischen Vorgehen bei Patienten mit chronischer Dyspnoe sind (im Gegensatz zu einer Vielzahl von Algorithmen bei verschiedenen chronischen Erkrankungen [16, 17]) nach Ansicht der Autoren nicht verfügbar.

Vor diesem Hintergrund haben wir in zwei Facharztpraxen Innere Medizin/Kardiologie einen einfachen diagnostischen Algorithmus zur Diagnostik bei Patienten mit chronischer Dyspnoe zur Anwendung gebracht. Dabei konnten wir zeigen, dass bei über 95\% aller Patienten die Ursache der chronischen Dyspnoe ausreichend geklärt werden konnte. Nach anamnestischer, klinischer und apparativer Diagnostik mittels EKG und transthorakaler Echokardiografie konnte bei mehr als 50\% und nach zusätzlicher Durchführung einer Bodyplethysmografie und Blutgasanalyse bei mehr als $80 \%$ der Patienten die Ursache der chronischen Dyspnoe bestimmt werden. Von den betreuenden Ärzten/innen wurde dann zur weiteren diagnostischen Abklärung eine Spiroergometrie durchgeführt, welche mehrheitlich die Ursache der chronischen Dyspnoe erkennen ließ.

Weiterführende Untersuchungen in Abhängigkeit des klinischen Bildes wurden im Ermessen der betreuenden Ärzte/innen nur in geringem Umfang als notwendig erachtet.

In der Literatur existieren seit mehreren Jahren Ansätze zur Differenzierung der Patienten mit akuter Dyspnoe anhand von lungenfunktionellen Werten, wobei häufig der PEF-Wert (entweder als Nominalwert bzw. in Prozent der Norm) genutzt wird. Die Rationale besteht darin, dass in den Studien dieser Wert immer signifikant geringer bei Patienten mit pulmonaler Ursache im Vergleich zu Patienten mit kardialer Dyspnoe zu finden war (z. B. $144 \pm 66$ vs. $267 \pm 97 \mathrm{~L} / \mathrm{min}$ [15], $108 \pm 49 \mathrm{~L} / \mathrm{min}$ vs. $224 \pm 82$ [13] bzw. 139 vs. 243 L/min [14]. Bei Verwendung des PEF-Wertes in Prozent der Norm konnte ähnliche signifikante Unterschiede ge- 
funden werden (26,4\% vs. 48,9\% [14] bzw. $35 \pm 17 \%$ vs. $58 \pm 19 \%$ [15]). In einer Arbeit [15] wurde der DDI verwendet, wobei auch hierbei ein signifikanter Unterschied ( $8,4 \pm 4,0$ vs. $18,4 \pm 7,9)$ zwischen pulmonaler bzw. kardialer Ursache der Dyspnoe zu finden war. In dieser Studie differenzierte ein DDI-Wert von 13 am besten mit einer Sensitivität von $82 \%$ und Spezifität von $74 \%$ zwischen beiden Ursachen der akuten Dyspnoe. Die anhand kleiner Patientenzahlen ermittelten Daten machen deutlich, dass bei akuter Dyspnoe ein PEF-Wert von $<150 \mathrm{~L} / \mathrm{min}$ bzw. ein DDI-Wert von unter 13 mit klinisch ausreichender Sicherheit eine pulmonale Ursache anzeigt.

Für unsere untersuchten Patienten mit chronischer Dyspnoe erwiesen sich jedoch die in der Literatur verwendeten Grenzwerte zur Differenzierung zwischen kardialen und pulmonalen Ursachen der Dyspnoe als nicht sinnvoll.

\section{Limitationen}

Entsprechend der personellen Ressourcen in den beiden Praxen erfolgte keine systematische Rekrutierung von Patienten mit chronischer Dyspnoe. Die im Vorfeld vereinbarten Kriterien für die ausreichende Klärung einer chronischen Dyspnoe, die eingesetzten apparativen Verfahren und deren stufenweise Anwendung entsprechen den klinischen Erfahrungen, wurden jedoch nicht wissenschaftlich validiert. Die Anwendung der Parameter zur Differenzierung der akuten Dyspnoe bei unseren Patienten mit chronischer Dyspnoe war wissenschaftlich eher zum Generieren von Hypothesen gedacht.

\section{Danksagung \\ $\nabla$}

Das Projekt wurde mit finanzieller Unterstützung durch die Firma Actelion Pharmaceuticals Deutschland GmbH durchgeführt.

\section{Interessenkonflikt}

$\nabla$

R. Ewert hat Erstattungen von Reisekosten und Teilnahmegebühren für Kongresse, Honorare für Vorträge und finanzielle Zuwendungen für die Teilnahme an klinischen Studien erhalten.

A. Obst, C. Bahr, C. Weirich, F. Herschel und A. Rink geben an, dass kein Interessenkonflikt besteht.

\section{Literatur}

1 Parshall MB, Schwartzstein RM, Adams $L$ et al. An Official American Thoracic Society Statement: Update on the Mechanisms, Assessment, and Management of Dyspnea. Am J Respir Crit Care Med 2012; 185: $435-452$

2 Ewert R, Winkler J, Opitz C. Luftnot: Erfassung, Graduierung und Differenzierung. Atemwegs- und Lungenkrankheiten 2012; 38: 128-136

3 Cullen DL, Rodak B. Clinical utility of measures of breathlessness. Respir Care 2002; 47: 986-993

4 Garin 0 , Ferrer M, Pont $A$ et al. Disease-specific health-related quality of life questionnaires for heart failure: a systematic review with meta-analysis. Qual Life Res 2009; 18: 71 - 85

5 Speich R. Dyspnoe, Objektivierung des Subjektiven oder die Quadratur des Zirkels. Pneumologe 2012; 9: 248-253

6 Ewert R, Gläser S, Koch B et al. Plötzliche Dyspnoe - Ein Beitrag zur Differenzialdiagnose. Pneumologe 2012; 9: 279-284

7 Balady GJ, Arena R, Sietsema K et al. Clinician's Guide to cardiopulmonary exercise testing in adults: a scientific statement from the American Heart Association. Circulation 2010; 122: 191 - 225

8 Köllner V. Psychosomatische Aspekte des Dyspnoe. Pneumologe 2012; 9: $271-278$

9 Charlson ME, Pompei P, Ales $K$ et al. A new method of classifying prognostic comorbidity in longitudinal studies: development and validation. J Chron Dis 1987; 40: 373-383

10 Jones NL, Makrides L, Hitchcock C et al. Normal standards for an incremental progressive cycle ergometer test. Am Rev Respir Dis 1985; 131: $700-708$

11 Mahler DA, Wells CK. Evaluation of clinical methods for rating dyspnea. Chest 1988; 93: 580-586

12 Brown LH, Gough JE, Seim RH. Can quantitative capnometry differentiate between cardiac and obstructive causes of respiratory distress? Chest 1998; 113: 323-326

13 McNamara RM, Cionni DJ. Utility of the peak expiratory flow rate in the differentiation of acute dyspnea. Cardiac vs pulmonary origin. Chest 1992; 101: 129-132

14 Gough JE, Brewer KL. Can peak expiratory flow measurements differentiate chronic obstructive pulmonary disease from congestive heart failure? Emerg Med Int 2012: e-Pub 2011, Oct 27

15 Ailani RK, Ravakhah K, DiGiovine B et al. Dyspnea differentiation index: A new method for the rapid separation of cardiac vs pulmonary dyspnea. Chest 1999; 116: $1100-1104$

16 Zeng $Q$ Jiang $S$. Update in diagnosis and therapy of coexistent chronic obstructive pulmonary disease and chronic heart failure. J Thorac Dis 2012; 4: 310-315

17 Kwo PY. Shortness of breath in the patients with chronic liver disease. Clin Liver Dis 2012; 16: 321 - 329 